\title{
Unusual features of long-range density fluctuations in glass-forming organic liquids: A Rayleigh and Rayleigh-Brillouin light scattering study
}

\author{
A. Patkowski, ${ }^{1,2}$ E. W. Fischer, ${ }^{1}$ W. Steffen,${ }^{1}$ H. Gläser, ${ }^{1}$ M. Baumann, ${ }^{1}$ T. Ruths,,${ }^{1}$ and G. Meier ${ }^{1,3}$ \\ ${ }^{1}$ Max Planck Institute for Polymer Research, Ackermannweg 10, 55128 Mainz, Germany \\ ${ }^{2}$ Institute of Physics, A. Mickiewicz, University, Umultowska 85, 61-614 Poznan, Poland \\ ${ }^{3}$ Institut für Festkörperforschung, Forschungszentrum Jülich, Postfach 1913, 52425 Jülich, Germany
}

(Received 2 January 2001; published 23 May 2001)

\begin{abstract}
A new feature of glass-forming liquids, i.e., long-range density fluctuations of the order of $100 \mathrm{~nm}$, has been extensively characterized by means of static light scattering, photon correlation spectroscopy and RayleighBrillouin spectroscopy in orthoterphenyl (OTP) and 1,1-di(4'-methoxy-5' methyl-phenyl)-cyclohexane (BMMPC). These long-range density fluctuations result in the following unusual features observed in a light scattering experiment, which are not described by the existing theories: (i) strong $q$-dependent isotropic excess Rayleigh intensity, (ii) additional slow component in the polarized photon correlation function, and (iii) high Landau-Placzek ratio. These unusual features are equilibrium properties of the glass-forming liquids and depend only on temperature, provided that the sample has been equilibrated long enough. The temperaturedependent equilibration times were measured for BMMPC and are about 11 orders of magnitude longer than the $\alpha$ process. It was found that the glass-forming liquid OTP may occur in two states: with and without long-range density fluctuations ("clusters"). We have characterized the two states by static and dynamic light scattering in the temperature range from $T_{g}$ to $T_{g}+200 \mathrm{~K}$. The relaxation times of the $\alpha$ process as well as the parameters of the Brillouin line are identical in both OTP with and without clusters. The $\alpha$ process (density fluctuations) in OTP was characterized by measuring either the polarized (VV) or depolarized (VH) correlation function, which are practically identical and $q$-independent. This feature, which is commonly observed in glass-forming liquids, is not fully explained by the existing theories.
\end{abstract}

DOI: 10.1103/PhysRevE.63.061503

PACS number(s): 78.35.+c, 64.70.Pf

\section{INTRODUCTION}

The question of whether the structure and dynamics in supercooled liquids are homogeneous or heterogeneous in space remains one of the most important unsolved problems in this field [1,2]. A large number of experimental studies clearly reveal the existence of dynamical heterogeneities [3-10]. In addition, early concepts for the explanation of the liquid to glass transition are based on the existence of cooperatively rearranging regions (CRR) $[11,12]$ and estimates of a characteristic length scale yield values of $2-3 \mathrm{~nm}$ near $T_{g}$ $[12,13]$. The same order of magnitude was found by spin diffusion NMR experiments [14]. In molecular-dynamics simulations for Lennard-Jones liquids, it has been shown that spatial correlations between the displacements of molecules become increasingly long on approaching the mode-coupling temperature $T_{c}$ [15]. Other simulations of monatomic liquids revealed that the number of icosahedral clusters increases with decreasing temperature [16].

The Rayleigh-Brillouin spectrum, i.e., the spectrum of density fluctuations for liquids whose molecules possess internal degrees of freedom that are weakly coupled to the translational degree of freedom of the fluid, has been calculated for simple liquids [17], relaxing liquids [18,19], and polymeric liquids [20]. The experimental features of the Brillouin doublet as a function of temperature for simple and polymeric glass-forming liquids are in a good agreement with the theoretical predictions, apart from some controversy concerning the distribution and temperature dependence of the structural relaxation time [21-23].
The main discrepancy between theory and experiment for glass-forming liquids is the intensity of the central Rayleigh line and the value of the Landau-Placzek ratio, $R_{\mathrm{LP}}$, i.e., the ratio of the integral intensities of the Rayleigh line and the Brillouin doublet. Experimental data obtained for simple and polymeric glass-forming liquids show very high values of the $R_{\mathrm{LP}}$ (higher than 10 and sometimes higher than 100) at low temperatures close to $T_{g}$ [24-27], while theories predict $R_{\mathrm{LP}}$ not higher than about 1.5. The discrepancy between the theory and experiment concerning the intensity of the central Rayleigh line is additionally corroborated by the angular dependence of the intensity of scattered light. These measurements reveal a high excess isotropic component that is increasing with decreasing scattering angle. This indicates the existence of long-range density fluctuations. No excess intensity was observed in the depolarized (VH) Rayleigh component. The correlation length in orthoterphenyl (OTP) amounted to $400 \mathrm{~nm}$ at low temperatures and it persisted even above $T_{m}$ [28]. This excess scattering, the high experimental values of the $R_{\mathrm{LP}}$, and an additional slow component (about seven orders of magnitude slower than the usual density fluctuations) in the density correlation function were found experimentally in other glass-forming liquids $[26,28,29]$. They seem to be general features of glassforming liquids and represent serious discrepancies with the theoretical picture. These unusual features can be explained by "dynamic clusters"' [24,30], i.e., long correlation length density fluctuations. The presence of the slow component in the PCS correlation functions leads to an apparent nonergodicity at temperatures close to $T_{g}$ [31]. Recently, it was found $[28,32]$ that besides the normally occurring OTP with clusters, also cluster-free OTP samples can be prepared by a 
special treatment. The cluster-free OTP behaves according to the theoretical predictions $[17,18]$ and does not show the unusual features that were observed in OTP with clusters as well as other glass-forming liquids.

The question arises of whether these long-range density fluctuations are an equilibrium phenomenon, i.e., the angular dependent intensity always reaches the same value regardless of the individual thermal history of the sample. We were able to prove in this paper at least for one substance [1,1-di (4'-methoxy-5' methyl-phenyl)-cyclohexane (BMMPC)] that the final intensity after some (temperature-dependent) equilibration time is not dependent on thermal history. A similar result was obtained for poly (methyl- $p$-tolyl-siloxane) PMpTS [29]. For OTP, it is known that long-range density fluctuations appear again at room temperature, when they have been suppressed before by keeping the sample at high temperatures $\left(T_{g}+150 \mathrm{~K}\right)$ [33] for several hours. The equilibrated samples have been analyzed by means of static light scattering (SLS) and photon correlation spectroscopy (PCS) to record the temperature- and angle-dependent intensity and correlation functions. The results of the PCS measurements can be connected with ideas of cooperative relaxation [34].

Recently, we have measured the structure factor of fragile glass-forming liquid, OTP, in a previously inaccessible $q$ range $\left(0.02-0.6 \mathrm{~nm}^{-1}\right)$ between the conventional LS and SAXS $q$ ranges using the ultralow angle $\mathrm{x}$-ray scattering (USAXS) beamline at ESRF [35]. At low $q$ (overlapping with the LS $q$ range), this structure factor exhibits an excess scattering consistent with the LS data. At high $q$ (overlapping with the SAXS-Kratky camera $q$ range) this structure factor decays to a plateau defined by the isothermal compressibility, in agreement with the previous SAXS studies [30]. Thus, the long-range density fluctuation in glassforming liquids can be observed both in the isotropic component of scattered light and in the USAXS, provided the proper $q$ range is covered. The power-law $q$ dependence of the scattered intensity in the intermediate $q$ range indicates that the excess scattering is not due to large bulky dense regions but to fractal aggregates of denser domains.

In this paper we will (i) study the equilibration times and equilibrium properties of long-range density fluctuations in BMMPC by means of SLS and PCS, and (ii) characterize the newly found cluster-free OTP and compare it with the usually studied OTP with clusters in terms of static Rayleigh light scattering, Rayleigh-Brillouin spectroscopic properties, and the characteristics of the primary $(\alpha-)$ relaxation process in the time range of photon correlation spectroscopy. The main objective of this paper is to present the experimental evidence for long-range density fluctuations (as they are seen in a light scattering experiment) which result in the unusual experimental features described above.

To our knowledge, there are no quantitative theories that describe these long-range density fluctuations, although a qualitative model has been proposed $[30,36]$ in which the long-range fluctuations are due to agglomeration of the cooperatively rearranging domains. The unusual experimental features described in detail in this paper for OTP and BMMPC have been observed in other low molecular weight and polymeric glass-forming liquids and are general features of this class of materials. Therefore, a complete theory of the glass transition should be able to describe the long-range density fluctuations and the unusual features resulting from them. Another description of the behavior of glass-forming liquids using two order parameters-density and bond order-has been proposed [37,38]. This model predicts the existence of long-range density fluctuations with all the features observed in our study.

The primary $(\alpha)$ process in glass-forming liquids in the theoretical description is discussed as being due to density fluctuations. Experimentally, however, it is often characterized by methods that are sensitive to the orientation of molecules, i.e., dynamic depolarized light scattering, dielectric relaxation, and NMR.

The density fluctuations can, in principle, be measured by means of photon correlation spectroscopy as a difference between the polarized $(\mathrm{VV})$ and depolarized $(\mathrm{VH})$ correlation functions: $g_{\text {iso }}(\tau)=g_{V V}(\tau)-\frac{4}{3} g_{\mathrm{VH}}(\tau)$. In practice, however (in the case of low molecular weight glass-forming liquids), these two correlation functions (and the correlation times obtained from them) are practically identical. This is the experimental justification to use the orientational relaxation times to characterize the density fluctuations, i.e., it is assumed that both processes have identical dynamics. This can be rationalized in the framework of the mode-coupling theory (MCT) [39], although the physical mechanisms of light scattering in these systems are complex [40,41].

\section{MATERIALS AND METHODS}

\section{A. Sample preparation}

Orthoterphenyl (OTP) was bought from MerckSchuchardt (99\% pure), several times recrystallized from methanol solution, and distilled under vacuum into dust-free light scattering cells (round 10-20-mm outer diameter). The purified OTP showed a melting temperature $T_{m}$ of $328.5 \mathrm{~K}$ and a glass-transition temperature [measured by means of differential scanning calorimetry (DSC) at a cooling rate of $10 \mathrm{~K} / \mathrm{min}$ ] amounting to $T_{g}=244.8 \mathrm{~K}$, in good agreement with the literature data [42]. The BMMPC samples were synthesized in our laboratory following the procedure given in Ref. [43]. The melting temperature of BMMPC amounted to $T_{m}=346 \mathrm{~K}$ and the glass-transition temperature, measured by means of DSC at a cooling rate of $10 \mathrm{~K} / \mathrm{min}$, amounted to $T_{g}=261 \mathrm{~K}$. The samples were filtered three times at $353 \mathrm{~K}$ through a $0.22-\mathrm{mm}$ Millipore filter into dust-free cylindrical cells made out of Pyrex tubes. Distillation of BMMPC into the light scattering cell was not possible due to decomposition of the sample beyond $370 \mathrm{~K}$, which is below the boiling point under high vacuum.

\section{OTP with and without clusters}

Cluster-free OTP is defined as a sample with constant scattered intensity at all scattering angles. In order to obtain a cluster-free sample, OTP was vacuum-distilled into dustfree light scattering cells using only the middle fraction. It was absolutely necessary to avoid any crystallization of OTP in the cell by keeping all the distillation equipment and the 


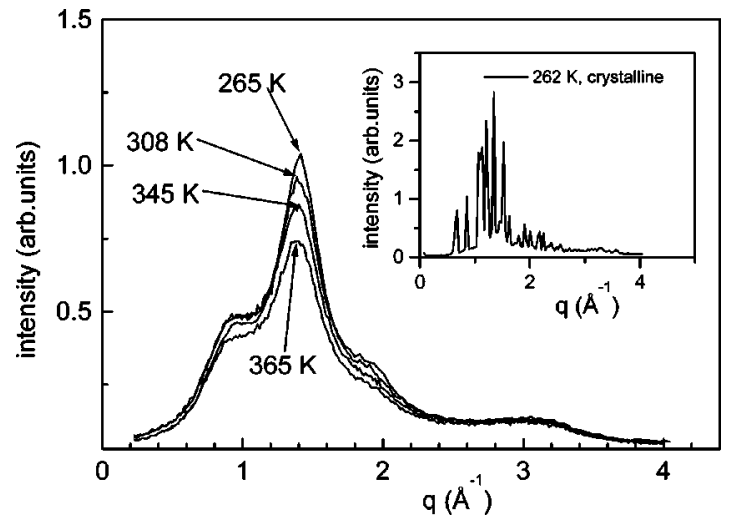

FIG. 1. Wide-angle x-ray scattering data (WAXS) measured for OTP for different temperatures above $T_{g}$. The corresponding data for a crystalline sample are shown in the inset.

cell at a temperature above $T_{m}$ during distillation. Even small traces of crystallization during the sample preparation resulted in OTP samples with clusters, even after annealing them after preparation for several hours above $T_{m}$. OTP samples with clusters were obtained by crystallizing OTP in the light scattering cells (cooling in liquid nitrogen followed by crystallization at room temperature) and then melting them at $344 \mathrm{~K}$ and cooling to room temperature. Thus the same sample may be prepared in the state with or without clusters using this procedure. The temperature of $344 \mathrm{~K}$ was maintained for several hours to be sure to leave no trace of crystallization. Wide-angle x-ray scattering (WAXS) has been measured for OTP samples with clusters (Fig. 1) in the temperature range of 265-365 K. No trace of crystallization can be seen in these data. The WAXS curves exhibit characteristic features observed also for other supercooled liquids [44]. OTP samples obtained in this way were characterized by means of measurements of the angular dependence of the scattered intensity. OTP with clusters showed increasing scattered intensities at decreasing scattering angles. From the angular dependence of the scattered intensity, the correlation length was calculated. Both samples, with and without clusters, showed no difference in their crystallization behavior. It was extremely difficult to achieve crystallization in the very pure samples because of the very low homogeneous nucleation rate [42]. It was possible to crystallize the sample only by introducing heterogeneous nucleation sites (cracks) by rapidly cooling the sample in liquid nitrogen.

\section{B. Experimental methods}

The experimental setup used to measure the RayleighBrillouin spectra was described in detail elsewhere [32]. A single-mode argon-ion laser (Spectra-Physics, Model 165) with a power of up to $400 \mathrm{~mW}$ at $\lambda=514 \mathrm{~nm}$ was used as the light source. The incident beam was polarized vertically by means of a Glan polarizer (Bernhard Halle, extinction coefficient better than $10^{-6}$ ). The vertical or horizontal polarization of the scattered light was selected by means of a GlanThompson polarizer (Bernhard Halle, extinction coefficient better than $10^{-7}$ ). A planar Fabry-Perot interferometer (FPI) was used (Burleigh, Model RC 10) of a free spectral range
(FSR) of $27 \mathrm{GHz}$ and a finesse better than 50 . The position of the Rayleigh line was stabilized during accumulation (1800 sweeps, $1 \mathrm{~s}$ each) by means of a DAS-10 system (Burleigh). The spectra were accumulated in a multichannel analyzer (Silena, Varro 16K) and then transferred to an IBM AT computer. Each spectrum consisted of three orders of FSR. The first order was the instrumental function and the two subsequent orders consisted of the spectra of the scattered light (for a detailed description, see Ref. [32]). The absolute scattered intensities were calculated using toluene as a standard. The Rayleigh ratio (isotropic) of $22.9 \times 10^{-6} \mathrm{~cm}^{-1}$ $(\lambda=488 \mathrm{~nm})$ [45], corrected for the wavelength of $514.5 \mathrm{~nm}$ $\left(18.5 \times 10^{-6} \mathrm{~cm}^{-1}\right)$, was used. The isotropic component of the Rayleigh-Brillouin spectrum was calculated using the relation $I_{\mathrm{iso}}(\omega)=I_{\mathrm{VV}}(\omega)-4 / 3 I_{\mathrm{VH}}(\omega)$ by a point by point subtraction of the polarized, $I_{\mathrm{VV}}(\omega)$, and depolarized, $I_{\mathrm{VH}}(\omega)$, Rayleigh-Brillouin spectra measured under identical experimental conditions. Two cell holders were used. (i) At temperatures from 293 to $433 \mathrm{~K}$, the sample was placed in a metal block with holes for the incident and scattered light; the temperature was controlled by electric heaters with an accuracy of $0.1 \mathrm{~K}$. (ii) In the temperature range from 233 to $293 \mathrm{~K}$, another cell holder with vacuum insulation was used in order to prevent the condensation of water on the light scattering cell. The temperature of the sample was controlled by a thermostat with circulating liquid with an accuracy of $0.2 \mathrm{~K}$. In the static light scattering experiment (SLS), it is important to measure the intensity of scattered light at as low $q$ values as possible. Low $q$-values can be achieved by an increase of the wavelength of incident light $\lambda_{0}$, and by decreasing the scattering angle $\theta$. Therefore, in our studies we used a krypton-ion laser (Spectra-Physics, model 2020, $\lambda_{0}$ $=647 \mathrm{~nm}$ ) and scattering angles from $7^{\circ}$ to $150^{\circ}$. In this way we were able to cover the $q$ range from $1.9 \times 10^{-3}$ to $2.8 \times 10^{-2} \mathrm{~nm}^{-1}$. For the SLS measurements, a modified optical setup [46] (ALV-Langen, FRG) was used in which a new cell housing was installed. The cell housing was made of glass ceramics (macor) and contained a double glass cylindrical window. The space between the glass cylinders was evacuated in order to increase the thermal insulation of the interior of the cell. In this way, the external glass cylinder was kept at room temperature while the sample could be cooled down to $230 \mathrm{~K}$ without any problems due to water condensation or temperature gradients. The intensity of scattered light was measured using a photon-counting system. The absolute Rayleigh ratio was calculated using toluene as a standard, with $R_{\text {tol }}=9.6 \times 10^{-6} \mathrm{~cm}^{-1}$ at $\lambda_{0}=647 \mathrm{~nm}$. To get rid of the speckles due to the slow process [31,47], the sample was rotated by a home-made device at frequencies from 0.5 to $5.5 \mathrm{rpm}$. The photon correlation spectroscopy (PCS) measurements were performed using the same optical setup as for the SLS. The correlation functions were measured using a digital correlator (ALV-5000, Langen, FRG).

\section{RESULTS AND DISCUSSION}

\section{A. Equilibration of BMMPC}

Temperature jump experiments have been performed to measure the kinetics of the formation of long-range density 


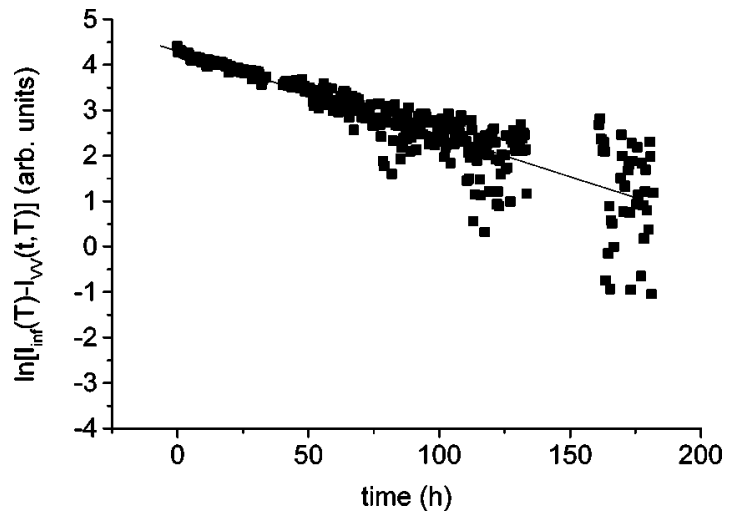

FIG. 2. Equilibration of BMMPC after a temperature jump from 343 to $313 \mathrm{~K}$ measured at $q=0.010 \mathrm{~nm}^{-1}$ in VV geometry. The straight line is the fit curve of an exponential function.

fluctuations. The BMMPC sample was kept at $343 \mathrm{~K}$ until the intensity at a given angle did not change any more. This was always achieved within several hours at this temperature. Then the sample was cooled down to a certain temperature within 2 min and the intensity was measured at an angle $\theta=40^{\circ}\left(q=0.010 \mathrm{~nm}^{-1}\right)$ as a function of time. In Fig. 2, the polarized (VV) intensity is shown as a function of tempering time after a temperature jump from $343 \mathrm{~K}$ to $313 \mathrm{~K}$. There is an exponential increase of intensity onto a saturation level according to the expression

$$
I_{\mathrm{VV}}(t, T)=I_{\infty}(T)-I_{\Delta}(T) \exp \left(-\frac{t}{\tau_{\text {equi }}(T)}\right),
$$

with $I_{\infty}(T)$ being the final (equilibrium) VV intensity, $I_{\Delta}(T)$ the intensity change due to the temperature jump, $t$ the tempering time, and $\tau_{\text {equi }}$ the equilibration time. Always the same $I_{\infty}(T)$ is reached regardless of from which temperature the temperature jump was started. After equilibration, the sample was heated again up to $343 \mathrm{~K}$ and the same procedure was repeated with another final temperature. The values obtained from the exponential fit for the different temperature jumps are listed in Table I.

In an Arrhenius plot of the equilibration times, the apparent activation energy is $123 \pm 16 \mathrm{~kJ} / \mathrm{mol}$. This unreasonably high value of the activation energy indicates that a VogelFulcher-Tamman (VFT) equation rather than the Arrhenius law should be used to describe this temperature dependence.

TABLE I. Equilibration times $\tau_{\text {equi }}$ obtained for BMMPC from temperature jump experiments from the initial temperature of $70^{\circ} \mathrm{C}$ to the final temperature $T_{f}$. The final absolute scattered intensities $R_{\infty}$ at equilibrium are also given.

\begin{tabular}{lcc}
\hline \hline$T_{f}(\mathrm{~K})$ & $R_{\infty}\left(10^{-6} \mathrm{~cm}^{-1}\right)$ & $\tau_{\text {equi }}(\mathrm{h})$ \\
\hline 343 & 39.2 & \\
333 & 54.4 & 4.35 \\
323 & 93.7 & 18.6 \\
318 & 102.7 & 52 \\
313 & 120.7 & 65 \\
\hline \hline
\end{tabular}

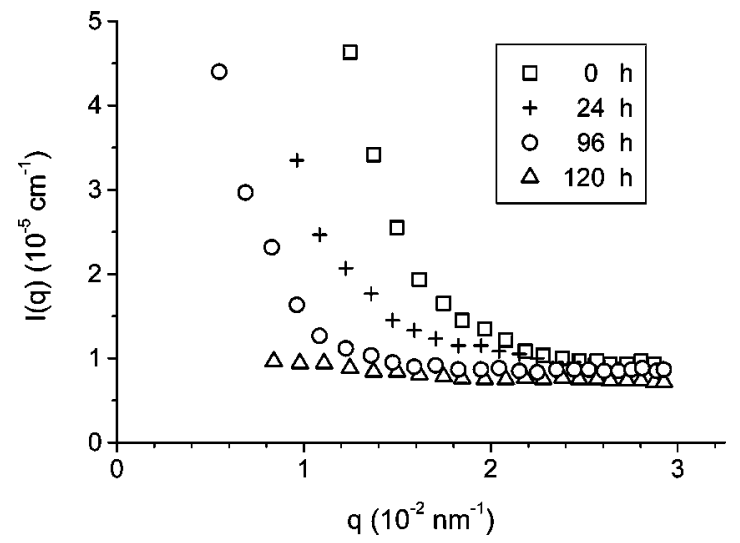

FIG. 3. Static light scattering curve $I(q)$ of OTP measured at $T_{g}+120 \mathrm{~K}$ after various annealing times at this temperature.

It cannot be decided whether the equilibration times behave according to an Arrhenius law or a Vogel-Fulcher-Tammann (VFT) law,

$$
\tau=\tau_{0} \exp \left(\frac{B}{T-T_{\infty}}\right)
$$

due to the limited temperature range of the measurements. From these temperature jump experiments one can conclude the following. (i) The long-range density fluctuations have an equilibrium state that is not dependent on the thermal history of the sample. (ii) The equilibration times increase drastically with decreasing temperature. (iii) The similarity of the equilibration times of the jumps back to $343 \mathrm{~K}$ show, that $\tau_{\text {equi }}$ mainly depends on the final temperature and not on the starting temperature. Similar results were obtained for a polymeric glass-forming liquid [29]. The equilibration process can also be described in the same way as the aggregation kinetics of a fractal cluster [48]. This approach will be discussed in a forthcoming paper [49]. The correlation length in OTP samples with clusters depends on temperature and their thermal history. By annealing a sample at high temperature above $T_{m}$, one can substantially reduce the excess scattering, which is decaying strongly with the annealing time [28], and obtain an apparently cluster-free sample, Fig. 3. The clusters will appear again in this sample upon cooling down, in contrast to the cluster-free samples, in which no clusters were observed even after very slow cooling (a few degrees per day) in the same temperature range.

\section{B. Static light scattering}

In order to characterize the long-range density fluctuations, the angular dependence of the isotropic Rayleigh component,

$$
R_{\mathrm{iso}}(\vec{q})=R_{\mathrm{VV}}(\vec{q})-\frac{4}{3} R_{\mathrm{VH}}(\vec{q})
$$

was measured. In the case of glass-forming liquids, it is generally observed that the isotropic Rayleigh component contains an additional strongly $q$-dependent component $R_{\text {iso }}^{\operatorname{exc}}(\vec{q})$ : 


$$
R_{\mathrm{iso}}(\vec{q})=R_{\mathrm{iso}}^{\prime}(\vec{q})+R_{\mathrm{iso}}^{\mathrm{exc}}(\vec{q})
$$

For simple (nonrelaxing) liquids, the $R_{\text {iso }}^{\prime}$ does not depend on the scattering angle. This angular independent isotropic component can be expressed in terms of the isothermal compressibility $\beta_{T}$ of the liquid,

$$
R_{\mathrm{iso}}^{\prime}=\frac{4 \pi^{2} n^{2}}{\lambda_{0}^{4}}\left[\rho \frac{\partial n}{\partial \rho}\right]^{2} k_{B} T \beta_{T},
$$

where $\lambda_{0}$ is the wavelength of light, $n$ is the refractive index, $\rho$ is the density, and $k_{B}$ is the Boltzmann constant. The anisotropic (depolarized) component depends on the effective optical anisotropy $\gamma$ of the molecules and it can be written as

$$
R_{\mathrm{VH}}=\frac{16}{15} \frac{\pi^{4}}{\lambda_{0}^{4}} \frac{\left(n^{2}+2\right)}{9} N \gamma^{2},
$$

where $N$ is the number of scatterers. In fluids, the value of the effective anisotropy depends on orientational correlations of the molecules. The angular dependence of the excess isotropic intensity can be described by an Ornstein-Zernike (OZ) formula,

$$
R_{\text {iso }}^{\operatorname{exc}}(\vec{q})=R_{\text {iso }}^{\text {exc }}(q=0) \frac{1}{1+\xi_{\mathrm{OZ}}^{2} q^{2}}
$$

where $\xi_{\mathrm{OZ}}$ is the correlation length of density fluctuations, $q$ is the scattering vector $\left[q=|\vec{q}|=\left(4 \pi n / \lambda_{0}\right) \sin (\Theta / 2)\right], n$ is the refractive index, $\lambda_{0}$ is the wavelength, and $\Theta$ is the scattering angle. To obtain Eq. (7), the density space correlation function was assumed to have the form

$$
g(r) \propto(1 / r) \exp \left(-r / \xi_{\mathrm{OZ}}\right) .
$$

In a more general form, the density autocorrelation function can be described by introducing a fractal dimension $D$ [51]:

$$
g(r)=\frac{a}{r^{3-D}} \exp \left(-\frac{r}{\xi}\right),
$$

with its Fourier transform,

$$
\begin{aligned}
S(q)= & \frac{4 \pi a}{q}\left(q^{2}+\xi^{-2}\right)^{(1-D) / 2} \Gamma(D-1) \\
& \times \sin (D-1) \arctan (q \xi)
\end{aligned}
$$

where $\Gamma(x)$ is the gamma function. The Ornstein-Zernike structure factor [Eq. (7)] is a special case with $D=2$ and the Debye correlation function $[50,55]$ is obtained for $D=3$. For the discussion of the experimental results (see Figs. 5 and 6 below), it may be useful to plot Eq. (10) in the form of the so-called Ornstein-Zernike plot: $1 / S(q)$ versus $q^{2}$ for various values of $D$ with a constant correlation length $\xi$, see Fig. 4. As one can notice, almost straight lines with different slopes are obtained, whereas for a constant $D=2$ and various val-

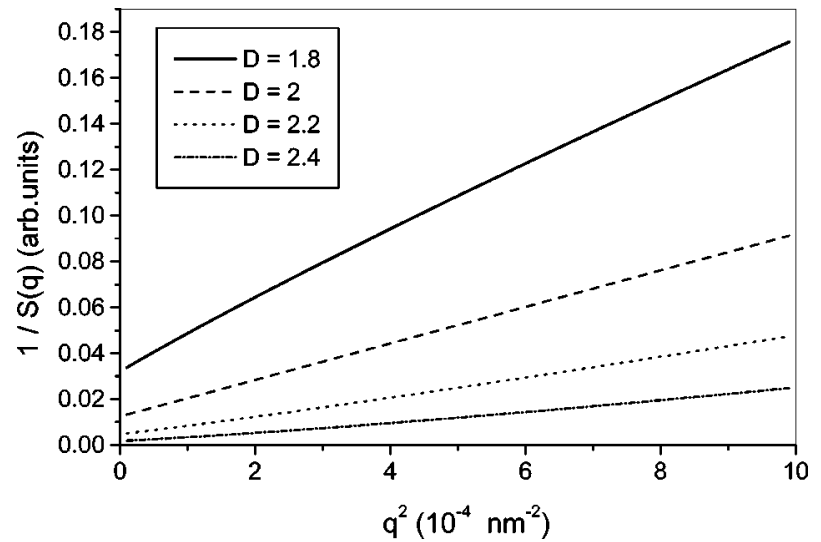

FIG. 4. Ornstein-Zernike plots of $1 / S(q)$ vs $q^{2}$ calculated using Eq. (10). The correlation length $\xi$ is kept constant, $\xi=80 \mathrm{~nm}$, and the fractal dimensions vary: $D=1.8$ (upper curve), 2.0, 2.2, and 2.4 (lower curve). $a=10^{-3}$.

ues of $\xi$, a family of parallel lines would result. Therefore, it is obvious that from light scattering data alone no clear conclusions concerning the values of $D$ and $\xi$ can be drawn. In order to obtain reliable data on $D$, the scattering experiment must cover a range $q>\pi / \xi$.

\section{Static light scattering on equilibrated BMMPC samples}

After equilibration of the samples according to the abovedescribed procedure, static measurements have been performed. The results are plotted in Fig. 5. The isotropic part of the VV intensity was computed using Eq. (3). The VH intensity remains independent of $q$, whereas the isotropic intensity is increasing with decreasing temperature and decreasing scattering angle. This angular dependence is due to long-range density fluctuations indicating that the state with long-range density fluctuations is the equilibrium state of the supercooled liquid. Figure 6 shows the isotropic intensities in an Ornstein-Zernike plot corresponding to the plot of Fig. 4. The inset shows the apparent temperature dependence of $\xi$

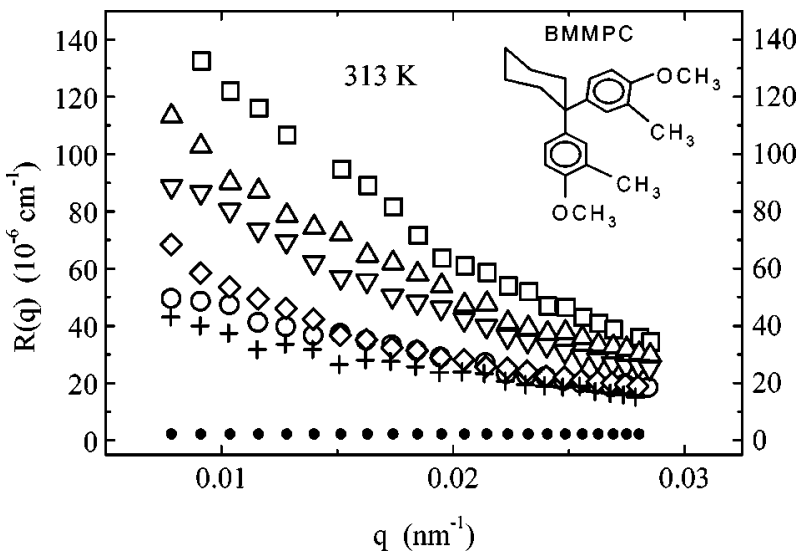

FIG. 5. Static measurement of equilibrated BMMPC at the temperatures $343 \mathrm{~K}(+), 333 \mathrm{~K}(\bigcirc), 328 \mathrm{~K}(\diamond), 323 \mathrm{~K}(\nabla), 318 \mathrm{~K}$ $(\triangle)$, and $313 \mathrm{~K}(\square)$. The isotropic intensity has been calculated using Eq. 3. Additionally, the intensity in VH geometry is plotted (O). 


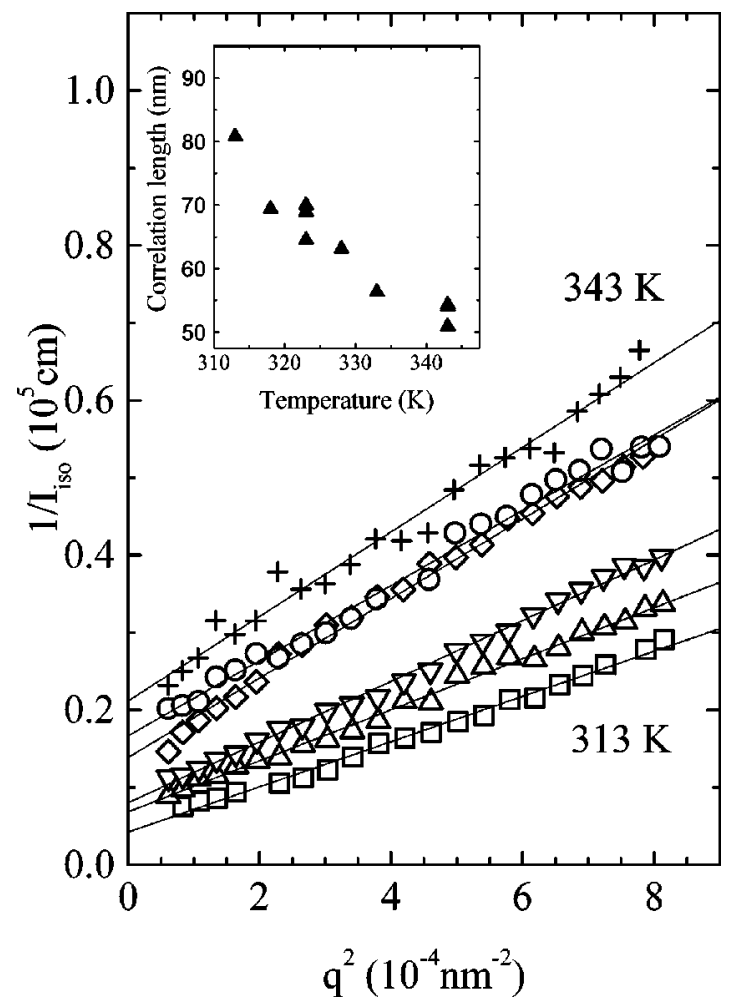

FIG. 6. Ornstein-Zernike plot of the isotropic intensities of an equilibrated sample of BMMPC at the temperatures $343 \mathrm{~K}(+), 333$ $\mathrm{K}(\bigcirc), 328 \mathrm{~K}(\diamond), 323 \mathrm{~K}(\nabla), 318 \mathrm{~K}(\triangle)$, and $313 \mathrm{~K}(\square)$. The inset shows the corresponding correlation lengths obtained from the linear regression curves.

under the assumption of $D=2$ [Ornstein-Zernike, Eq. (7)]. The fitting curves are not parallel, however, so one may conclude that the fractal dimension $D$ is also a function of temperature in that sense, and that $D$ increases with decreasing temperature. Due to the limited $q$ range, the data do not allow us to distinguish between density autocorrelation functions with different $D$ 's. Nevertheless, the strong increase of $\xi$ with decreasing temperature, as shown in Fig. 6 is only slightly changed by variation of $D$, as a closer inspection of Eq. (10) reveals.

\section{Static light scattering of OTP}

Practically all glass-forming liquids exhibit long-range density fluctuations (clusters), which seem to be a quasiequilibrium property of the supercooled liquids. This property, and the unusual experimental features related to it, are not predicted by any theory. On the other hand, the experimental features of the cluster-free OTP, which seems to be a quenched state of the supercooled liquid, are in agreement with the theories. Therefore, it is interesting to compare the light scattering properties of these two forms of OTP. The angular dependence of the measured polarized VV and depolarized VH components for OTP samples with and without clusters is shown in Fig. 7. As one can see, the cluster-free OTP behaves like a simple liquid (constant intensity), while for the OTP sample with clusters a strong angular-dependent

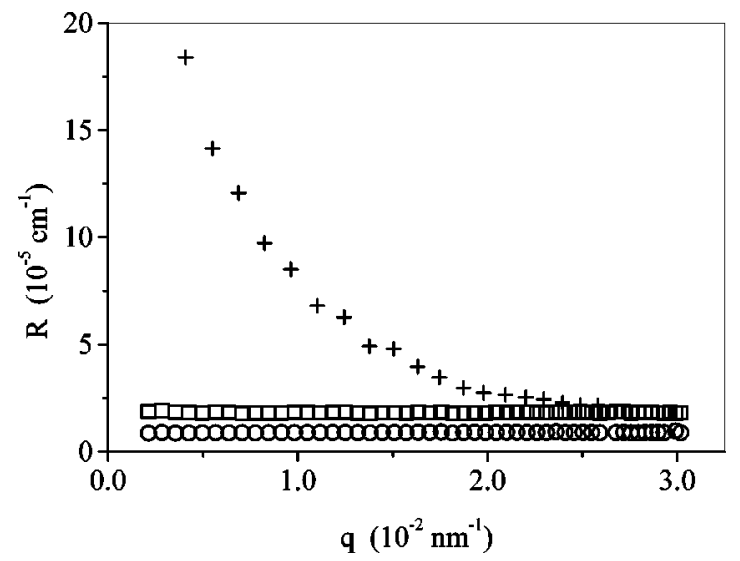

FIG. 7. Angular dependence of the polarized (VV) Rayleigh component for OTP samples with $(+)$ and without $(\square)$ clusters. The depolarized $(\mathrm{VH})$ component $(\mathrm{O})$ is identical for both samples.

excess scattering appears in the VV component. Since the depolarized components of scattered light are identical for both OTP with and without clusters, this excess scattering must be due to the isotropic contribution. Recently, we have shown [35] that the $q$ range between light scattering and conventional small angle $\mathrm{x}$-ray scattering (SAXS) can be covered by the ultrasmall-angle x-ray scattering (USAXS), which is now available at the European Synchrotron Radiation Facility at Grenoble. Figure 8 shows the combined structure factor obtained from the light scattering, USAXS, and SAXS data for OTP at $298 \mathrm{~K}$. The fit of Eq. (10) reveals a fractal dimension of $D=2.24 \pm 0.05$ and a correlation length of $\xi=154 \pm 20 \mathrm{~nm}$. In addition, an almost constant "tail" for $q>0.2 \mathrm{~nm}^{-1}$ is observed that is due to the classical density fluctuations proportional to the isothermal compressibility [35]. The excess scattering intensity caused by the long-range density fluctuations yields an extrapolated structure factor $\ln S(q \rightarrow 0) \cong 1.2$, which is about three orders of magnitude larger than $S(q=0)$ expected for a simple liquid on the basis of the compressibility relation [Eq. (5)].

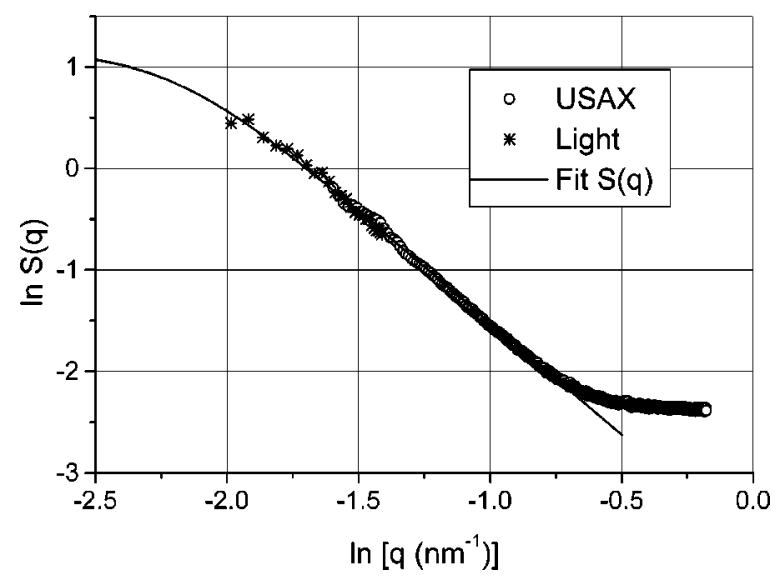

FIG. 8. USAXS and light scattering data of OTP at $298 \mathrm{~K}$ plotted in a double logarithmic scale. The solid line represents a fit using Eq. (10) with fit parameters $D=2.24 \pm 0.05, \xi=154 \pm 20 \mathrm{~nm}$, and extrapolated $\ln S(q=0)=1.18 \pm 0.25$. 


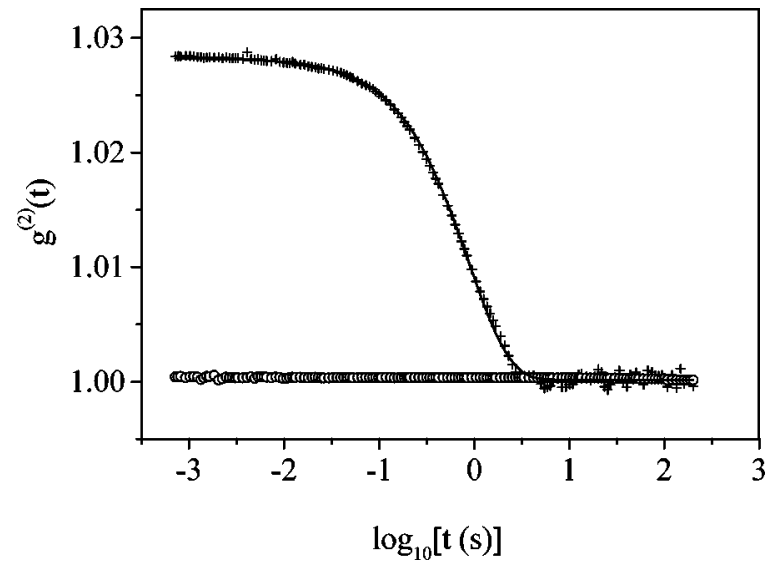

FIG. 9. Polarized $(\mathrm{VV})(+)$ and depolarized $(\mathrm{VH})(\mathrm{O})$ correlation function $g^{(2)}(t)$ measured for OTP with clusters at $293 \mathrm{~K}$. The solid line represents a single exponential fit to the data.

\section{The ultraslow mode}

Another striking difference between OTP samples with and without clusters is the presence of an additional slow component in the polarized (VV) photon correlation function for OTP with clusters, which does not appear in the corresponding correlation function for cluster-free OTP. The depolarized $(\mathrm{VH})$ correlation function does not contain this slow contribution. Such a slow process is not predicted by any theory of liquids. Both VV and VH correlation functions measured for OTP with clusters are shown in Fig. 9. The characteristic features of this slow process are that [43] (i) it can be described by a single exponential correlation function, and (ii) the correlation time is $q$-dependent, i.e., $1 / \tau \propto q^{2}$ for small $q$ 's. The features of the ultraslow mode of equilibrated samples are qualitatively the same as in nonequilibrated samples. Fitting the intensity correlation function of the slow mode according to the Kohlrausch-Williams-Watts formula,

$$
g^{(2)}(t)=1+\left|\alpha \exp \left[-\left(\frac{t}{\tau}\right)^{\beta}\right]\right|^{2},
$$

yields a $\beta$ parameter around 0.98 , i.e., the slow mode is practically single-exponential. As can be seen in Fig. 10, the amplitudes $[\alpha$ in Eq. (11)] and the correlation times are strongly $q$-dependent. Under the assumption that the mobility $\mu$ does not depend on frequency, the relaxation rate of the density fluctuations may be written as [53]

$$
\Gamma(q)=\frac{1}{\tau(q)}=\frac{\mu}{S(q, t=0)} q^{2},
$$

which yields approximately with the Ornstein-Zernike Eq. (7)

$$
\frac{1}{\tau(q)}=C q^{2}\left(1+q^{2} \xi^{2}\right),
$$

with $C$ being a constant depending on temperature and annealing conditions. Accordingly, Fig. 11 shows a slightly curved dependence of $1 / \tau$ versus $q^{2}$, although the $q$ range is

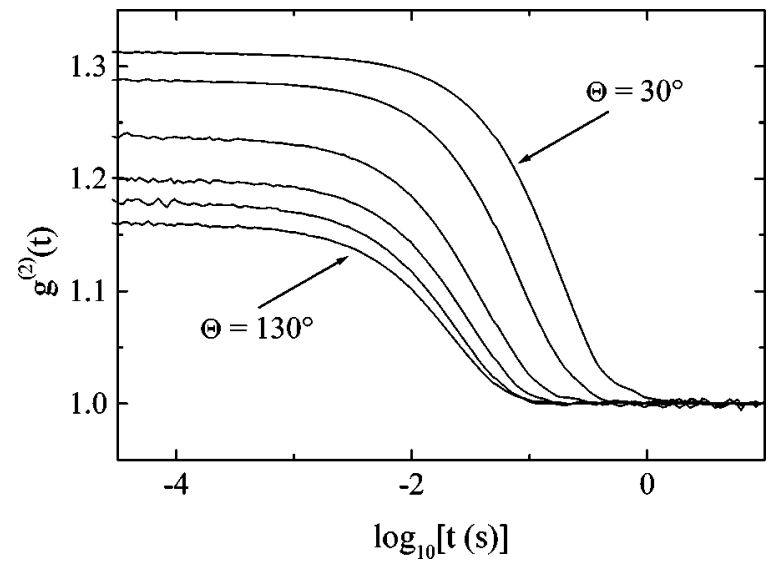

FIG. 10. The slow mode of equilibrated BMMPC at $354 \mathrm{~K}$ measured at $\Theta=30^{\circ}, 40^{\circ}, 60^{\circ}, 70^{\circ}, 110^{\circ}$, and $130^{\circ}$ increasing from the right- to the left-hand side.

too small for a precise determination of $\xi$. It may be mentioned that small-angle dynamic light scattering measurements down to $q^{2} \cong 10^{-6} \mathrm{~nm}^{-2}$ supported strongly Eq. (13) and yielded $\xi$ values, in good agreement with the static light scattering results [54].

Obviously, the heterogeneities causing the excess intensity [55] give rise to an apparent nonergodicity of the correlation function of the $\alpha$ process as discussed in detail in Ref. [31], and the ultraslow process reestablishes the ergodicity at much longer times. The temperature dependence of the relaxation time of the ultraslow process was studied in three glass-forming liquids: OTP, Salol, and BMMPC. The samples were cooled down and measured in a time interval much shorter than the equilibration time of the clusters, so that the cluster size did not change during the experiment. In Fig. 12, the temperature dependence of the relaxation time of the ultraslow process and the $\alpha$-peak position measured by means of dielectric spectroscopy are shown for comparison. As one can see the relaxation time of the ultraslow mode is proportional to the relaxation time of the $\alpha$ process in the entire temperature range studied. This means that the temperature dependence of the ultraslow mode can be described

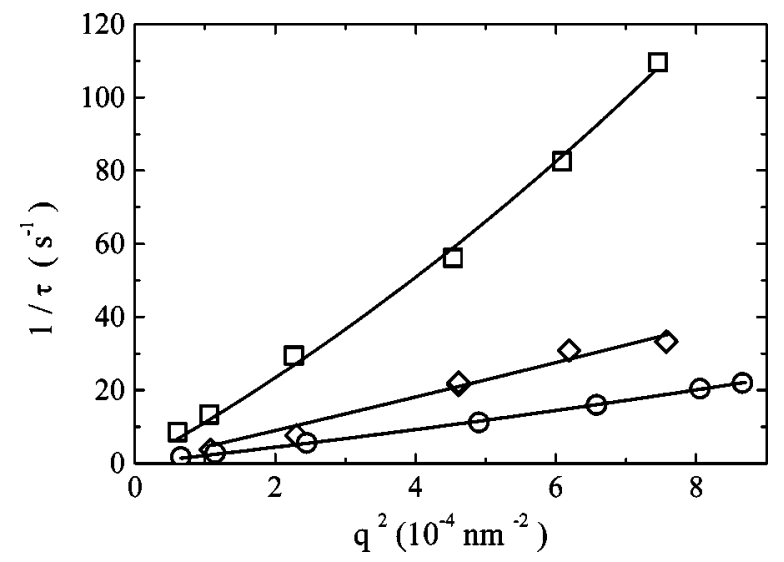

FIG. 11. $q^{2}$ dependence of the slow mode measured at $354 \mathrm{~K}$ for the substances BMMPC $(\square), \quad$ BMPC $\left[1,1^{\prime}\right.$-di $\left(4^{\prime}\right.$-methoxy-phenyl)-cyclohexane $](\diamond)$, and OTP $(\bigcirc)$. 


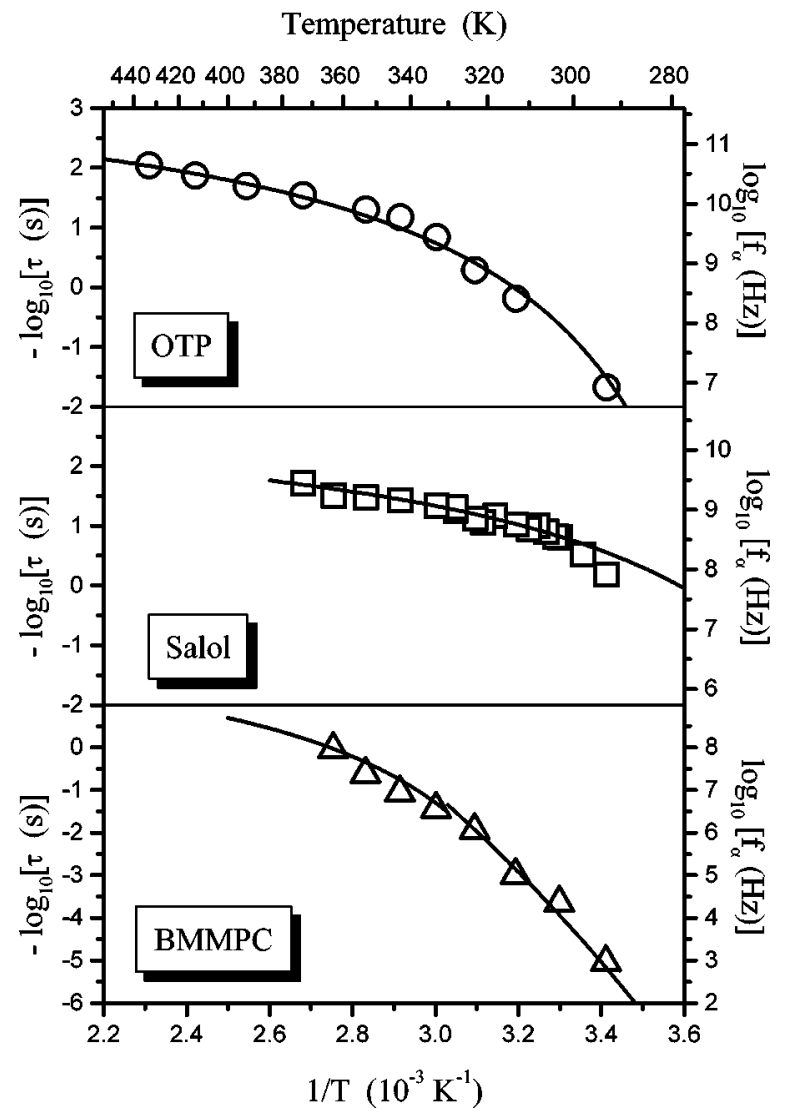

FIG. 12. Temperature dependence of the relaxation time of the ultraslow process in nonequilibrated samples of OTP, $\bigcirc$; Salol, $\square$; and BMMPC, $\triangle$. The solid lines represent the position of the $\alpha$ peak measured by means of the dielectric spectroscopy.

by a VFT formula with the same values of the parameters $B$ and $T_{\infty}$ as for the $\alpha$ process (viscosity) but with a different $\tau_{0}$. Thus we can conclude that the temperature dependence of the ultraslow process in samples with constant cluster correlation length results from the changes of the primary $(\alpha-)$ relaxation time and the corresponding changes of viscosity. All the results discussed above lead to the following physical picture of the long-range density fluctuations (clusters): The clusters are regions of higher density in a supercooled liquid. The ultraslow process, which has all the features of translational diffusion, can be assigned to a diffusion of the clusters due to diffusion of their boundaries. This diffusion of the cluster boundaries is due to a local growth and "melting" of the cluster on its surface without any mass transport involving the inner parts of the cluster and keeping the correlation length of the cluster constant at a constant temperature.

\section{Rayleigh-Brillouin spectra}

In order to compare the spectral features of OTP with and without clusters, the Rayleigh-Brillouin spectra were measured in the temperature range from 230 to $410 \mathrm{~K}$ for both samples. From the analysis of the isotropic RayleighBrillouin spectra, the Brillouin shift $\omega_{B}$, the Brillouin linewidth $\Gamma_{B}$, and the intensities of the Brillouin and the central Rayleigh lines have been obtained. The comparison of the

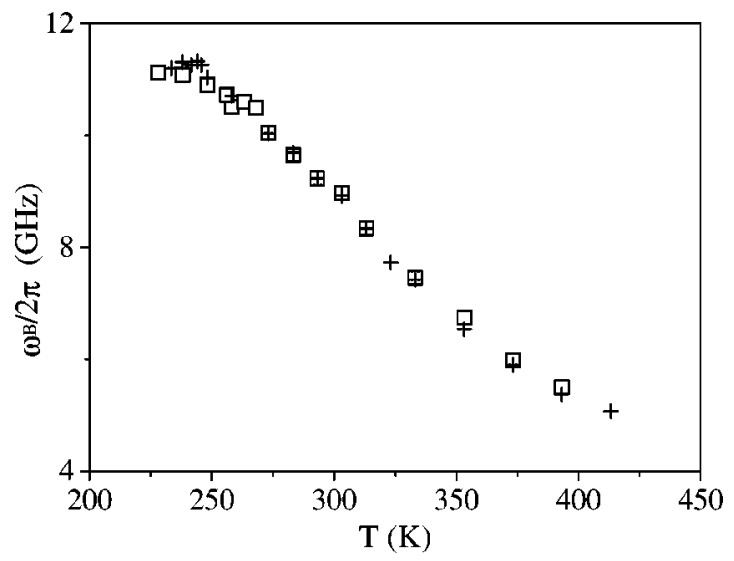

FIG. 13. Temperature dependence of the Brillouin shift $\omega_{B}$ for OTP with $(+)$ and without $(\square)$ clusters.

temperature dependence of the Brillouin shift $\omega_{B}$ for OTP with and without clusters is shown in Fig. 13. The values of $\omega_{B}$ obtained for OTP with and without clusters are identical within the experimental error in the entire temperature range studied, indicating identical longitudinal sound velocities in both samples. Our experimental results are in good agreement with previously published data $[24,57]$. The temperature dependence of the Brillouin linewidth $\Gamma_{B}$ for OTP with and without clusters is compared in Fig. 14. The values of $\Gamma_{B}$ for OTP with and without clusters are very similar in the entire temperature range studied. For both samples, a maximum of $\Gamma_{B}$ at about $355 \mathrm{~K}$ was found. Our $\Gamma_{B}$ data are also in good agreement with previously published results [24,57]. The features of the Brillouin spectrum of OTP are the same as the general features observed for viscoelastic liquids [5761]. The temperature dependence of the sound attenuation coefficient $\alpha \propto \Gamma_{B} / \omega_{B}$ is practically identical for OTP samples with and without clusters, and it looks very similar to Fig. 14. According to the classical acoustic theory [60], damping of a sound wave in a condensed medium with a single relaxation time $\tau_{d}$ should result in a maximum of $\alpha$ at a temperature at which $\tau_{d} \omega_{B} \cong 1$. Indeed, we have obtained such a maximum of the sound attenuation coefficient at about $355 \mathrm{~K}$ and a corresponding structural relaxation time $\tau_{d}$ at this temperature amounts to $25 \mathrm{ps}$. This relaxation time

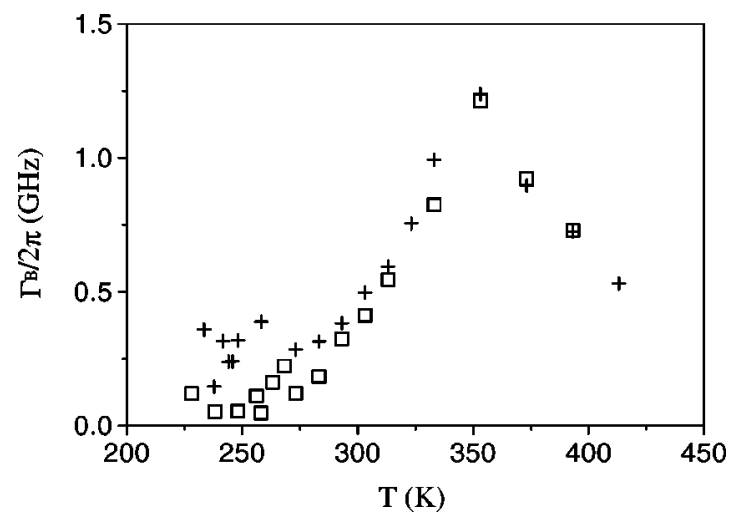

FIG. 14. Temperature dependence of the Brillouin line width $\Gamma_{B}$ for OTP with $(+)$ and without $(\square)$ clusters. 


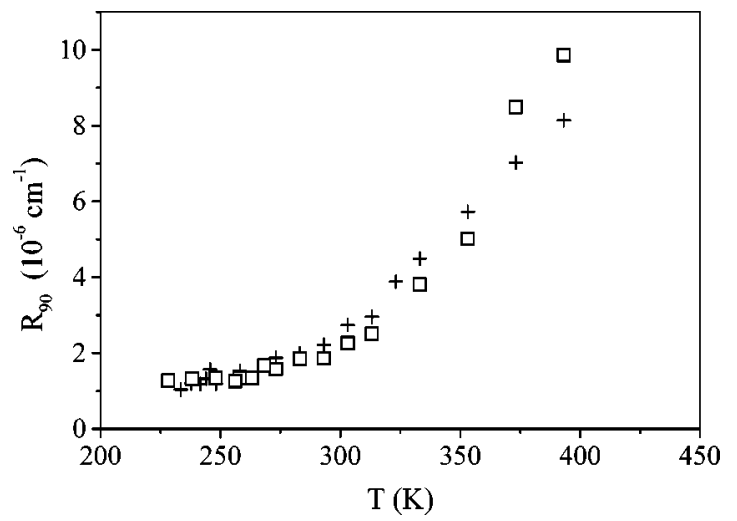

FIG. 15. Temperature dependence of the Brillouin intensity for OTP with $(+)$ and without $(\square)$ clusters.

$\tau_{d}$ is shorter by a factor of 6 than the relaxation time of the $\alpha$ process [32]. Such disagreement is usually observed in glassforming liquids and can be explained by new models of the Rayleigh-Brillouin spectra [62]. The integrated intensity of the Brillouin doublet can be expressed in terms of the adiabatic compressibility $\beta_{S, \omega_{B}}[52,63]$ measured at a frequency $\omega_{B}$ :

$$
I_{B}=\frac{\pi^{2}}{\lambda_{0}^{4}} \rho_{0}^{2}\left[\frac{\partial \varepsilon}{\partial \rho}\right]^{2} \beta_{S, \omega_{B}} k_{B} T
$$

The temperature dependence of this intensity for OTP with and without clusters is identical, as shown in Fig. 15 and in agreement with the value calculated from the Brillouin shift using Eq. (19). This indicates that the presence of clusters has no effect on the Brillouin spectrum, i.e., on the Brillouin shift, linewidth, and intensity.

\section{E. Landau-Placzek ratio}

The Landau-Placzek ratio, $R_{\mathrm{LP}}$, of OTP with and without clusters, obtained from the isotropic Rayleigh-Brillouin spectra, was measured at a scattering angle of $90^{\circ}$ using the planar FPI of FSR $=27 \mathrm{GHz}$ and a finesse of about 50. The temperature dependence of the $R_{\mathrm{LP}}$ (Fig. 16) is very different

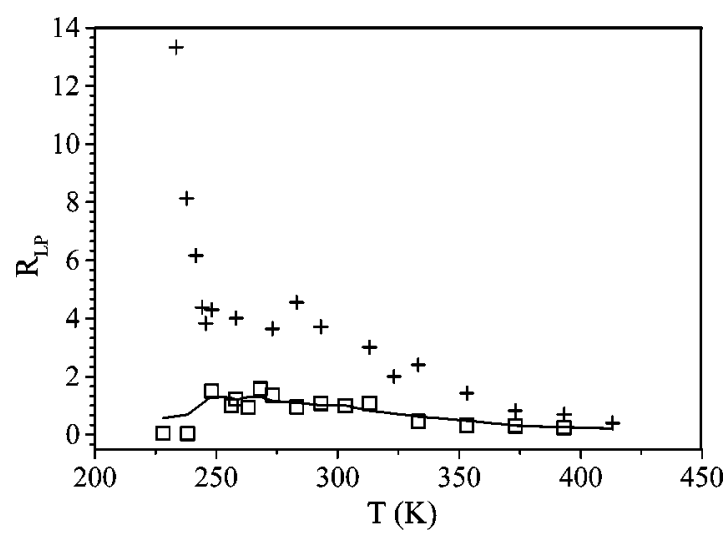

FIG. 16. Temperature dependence of the Landau-Placzek ratio $R_{\mathrm{LP}}$ for OTP samples with (+) and without $(\square)$ clusters. The solid line was calculated using Eq. (18). for OTP samples with and without clusters. For cluster-free OTP, the value of $R_{\mathrm{LP}}$ remains low in the entire temperature range studied, in contrast to the OTP sample with clusters, where the $R_{\mathrm{LP}}$ was increasing strongly with decreasing temperature, as reported previously $[24,25]$. The $R_{\mathrm{LP}}$ was theoretically calculated for relaxing liquids $[18,19,63]$ and can be expressed in the form

$$
R_{\mathrm{LP}}=\frac{\beta_{T, 0}}{1 / M^{\prime}\left(\omega_{\rho}\right)}-1=\frac{\gamma_{0} M^{\prime}\left(\omega_{\rho}\right)}{M_{0}}-1
$$

where $\beta_{T, 0}$ is the zero frequency isothermal compressibility, $M^{\prime}(\omega)$ is the real part of the longitudinal modulus of elasticity at a frequency $\omega_{p}$ equal to the Brillouin shift, and $\gamma_{0}$ $=C_{P} / C_{V}$ is the specific-heat ratio. We can write [19]

$$
M^{\prime}\left(\omega_{p}\right)=\rho_{0}\left[\frac{\omega_{p}}{q}\right]^{2}(1+2 \delta) \cong \rho_{0} v^{2},
$$

where $\rho_{0}$ is the average density, $\omega_{p} / q=v, v$ being the longitudinal sound velocity at the frequency $\omega_{p}$, and $q$ is the scattering vector. The correction $\delta$ is usually very small and can be neglected [19]. $M_{0}$ is the adiabatic bulk modulus

$$
M_{0}=\rho_{0} v_{0}^{2},
$$

where $v_{0}$ is the low-frequency longitudinal sound velocity. Thus, one obtains approximately

$$
R_{\mathrm{LP}} \cong \gamma_{0} \frac{v^{2}}{v_{0}^{2}}-1
$$

The solid line in Fig. 16, which represents a plot of Eq. (18) is in very good agreement with the experimental values of the $R_{\mathrm{LP}}$ for the cluster-free OTP. The sound velocity was taken from the measured Brillouin shift (cf. Fig. 13) and $v_{0}$ from ultrasonic measurements [64]. The temperature dependence of the $R_{\mathrm{LP}}$ for cluster-free OTP above $T_{g}$ is consistent with theoretical predictions for relaxing liquids $[18-20,63]$. Similar behavior of the $R_{\mathrm{LP}}$ was observed previously for glycerol [63]. Near the glass-transition temperature $T_{g}$, a drop in the calculated curve occurs because the lowfrequency sound velocity $v_{0}$ makes a jump in the direction of the high-frequency value $v_{\infty}$ of the glass. So the values of $v\left(\omega_{p}\right)$ and $v_{0}$ (glass) approach each other, leading to a lower value of the $R_{\mathrm{LP}}$. Such a decrease of the $R_{\mathrm{LP}}$ below $T_{g}$ was observed for the cluster-free OTP sample. A similar decrease was also observed for poly(methylmethacrylate) (PMMA) in our laboratory. In OTP samples with clusters, this effect is masked by the large excess intensity due to the long-range density fluctuations at low temperatures. These fluctuations are characterized by the long relaxation times of the ultraslow modes and are completely hidden in the central component $I(\omega \cong 0)$ of the Rayleigh-Brillouin spectrum. They behave like static inhomogeneities. As one can see in Fig. 16 , these contributions increase with falling temperature and there is no break or any discontinuity at the glass transition temperature. At higher temperatures, the measured $R_{\mathrm{LP}}$ of 
the "normal" OTP approaches the value of the cluster-free sample and the theoretical value according to Eq. (18).

The values of the $R_{\mathrm{LP}}$ for OTP with clusters were reproducible in a heating-cooling cycle at intermediate temperatures between 290 and $340 \mathrm{~K}$. Heating of the sample at higher temperatures for several hours resulted in a substantial reduction of the amount of clusters, which was not reversible in the short time (a few hours) of the experiment. The absolute values of the $R_{\mathrm{LP}}$ at temperatures below $293 \mathrm{~K}$ depend on the thermal history of the sample. The high values of the $R_{\mathrm{LP}}$ for OTP with clusters correlate well with the static light scattering results, i.e., in every case when high $R_{\mathrm{LP}}$ was obtained, the $R_{\text {iso }}(\vec{q})$ showed a strong excess scattering at low angles, indicating a high amount of clusters. Thus we can conclude that the high values of the $R_{\mathrm{LP}}$ for OTP with clusters are due to the presence of long-range density fluctuations. As far as the $R_{\mathrm{LP}}$ ratio is concerned, it is important to stress two points. (i) The value of the $R_{\mathrm{LP}}$ measured at a given temperature is not an equilibrium value but corresponds to a transient state and depends very strongly on the thermal history of the sample. (ii) The high value of the $R_{\mathrm{LP}}$ and its temperature dependence can be explained by the excess isotropic Rayleigh intensity. Due to very long equilibration times of clusters in OTP, this intensity is not changing much with temperature and does not follow the temperature dependence of the Brillouin intensity.

\section{F. Isothermal and adiabatic compressibilities}

In order to analyze the static light scattering data using any model, one has to know exactly the value of the angularindependent background that is subtracted from the angulardependent isotropic intensity. This background, which is due to density fluctuations is equal to the angular-independent isotropic intensity measured for cluster-free OTP. From this intensity the isothermal compressibility can be calculated using Eq. (5). The adiabatic compressibility can be calculated from the shift $\omega_{B}$ of the Brillouin line using the formula

$$
\beta_{S}=\frac{1}{\rho_{0} v^{2}}=\frac{1}{\rho_{0}\left(\omega_{B} / q\right)^{2}},
$$

where $\rho_{0}$ is the density, $v$ the sound velocity, and $q$ the scattering vector. Experimental values of the isothermal and adiabatic compressibility $\beta_{T}$ and $\beta_{S}$ of OTP obtained from our light scattering measurements at different temperatures are shown in Fig. 17 and compared with the isothermal compressibilities obtained from pressure-volume-temperature measurements [56] and small-angle x-ray scattering [30] experiments. The values of the compressibilities of OTP presented in this paper are comparable to those obtained previously [33] for annealed samples with clusters. The adiabatic compressibility $\beta_{S}$ is approaching the isothermal compressibility $\beta_{T}$ at high temperatures, leading to low LandauPlaczek ratios in agreement with our experiment, Fig. 16. The values of the isothermal compressibilities obtained from the total intensity measured by means of the static light scattering $\beta_{T}(\mathrm{SLS})$, integrated Rayleigh-Brillouin spectra $\beta_{T}$

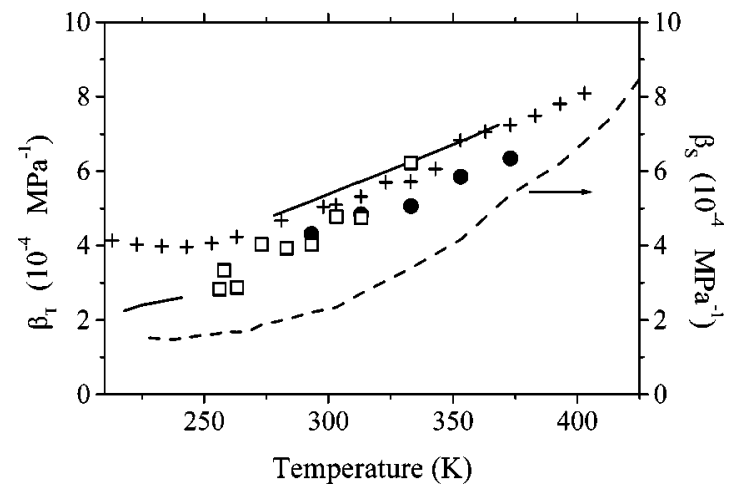

FIG. 17. Temperature dependence of the adiabatic compressibility calculated from the Brillouin shift $\beta_{S}$ (broken line) and the isothermal compressibility $\beta_{T}$ obtained from $\bullet$, static light scattering; $\square$, Rayleigh-Brillouin intensities; -, PVT from Ref. [54]; and + , small-angle $x$-ray scattering.

(RBS), small-angle x-ray scattering $\beta_{T}$ (SAXS), and PVT $\beta_{T}$ (PVT) are in reasonable agreement above $T_{g}$.

\section{G. Physical nature of clusters}

In our previous paper [35], we have shown that the excess scattered intensity exhibits a power-law $q$ dependence in a $\log$-log plot of intensity versus $q$. In this case (OTP), the power-law extended over a substantial $q$ range and could be easily identified, because the experimental data covered unusually broad $q$ range of almost two decades due to a combination of the SLS and ultrasmall-angle x-ray scattering (USAXS) data. The USAXS experiment has been performed at the ESRF, Grenoble. In all other cases, the USAXS data are not available, so this kind of analysis has to be performed using SLS data covering a much narrower $q$ range. In such a case, the power-law behavior can be observed only if the condition $\xi q>1$ is fulfilled over most of the $q$ range available, i.e., if the correlation length is sufficiently large. Such analysis based on the SLS data has been previously performed for aggregating silica colloids [65] where the powerlaw behavior and the fractal character of the aggregates have been observed. Figure 18 shows the log-log plot of the scattered isotropic intensity versus $q$ for PMpTS at different temperatures. As one can see, a substantial part of the data fol-

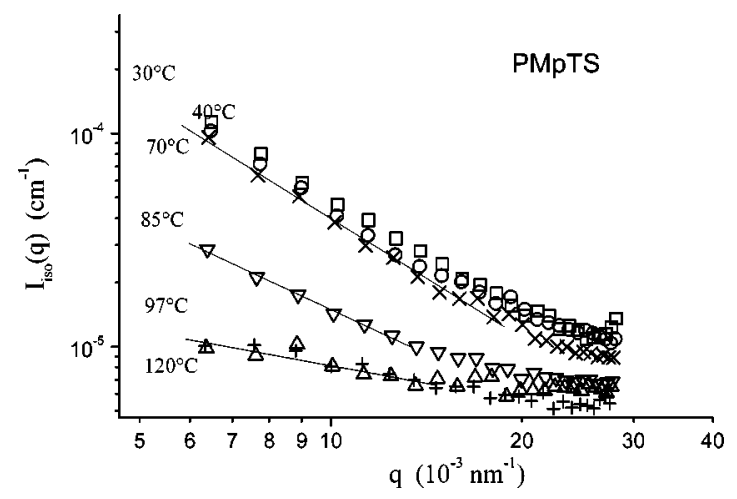

FIG. 18. A plot of $\log _{10} I_{\text {iso }}(q)$ versus $\log _{10} q$ for PMpTS at temperatures of $30,40,70,85,97$, and $120^{\circ} \mathrm{C}$ (top to bottom). 
lows the power law. Additionally, the absolute value of the fractal exponent (slope) is increasing with decreasing temperature, indicating an increase of the density of the fractal clusters (see [66]). We have shown previously [29] that the cluster size in PMpTS also increases with decreasing temperature. Thus, in PMpTS both the cluster size and density (fractal dimension) of the fractal clusters increase with decreasing temperature above $T_{g}$. Similar behavior has been observed in our laboratory for other glass-forming liquids. Thus, we can conclude that the long-range spatial heterogeneities in glass-forming liquids can be observed as an excess isotropic scattering due to long-range density fluctuations. Those heterogeneities (clusters) can be modeled as fractal aggregates of more dense regions. With decreasing temperature (above $T_{g}$ ), both the correlation length and the fractal dimension of these fractal clusters increase. The temperature dependence of the volume fraction of the denser regions can be described by the heterophase fluctuation model [36].

\section{CONCLUSIONS}

In a light scattering experiment, all glass-forming liquids exhibit strong discrepancies with the theories in terms of the Landau-Placzek ratio (too high), additional isotropic excess Rayleigh intensity, and an additional ultraslow component in the VV correlation function. These unusual features are due to the isotropic Rayleigh component, since the depolarized spectrum and the Brillouin lines behave as predicted by the theories. These features can be explained by spatial inhomogeneities or long-range density fluctuations (clusters), which are quasiequilibrium properties depending only on temperature, provided that the sample has been equilibrated long enough. The usually studied OTP with clusters was compared with a new cluster-free OTP obtained by a special treatment. The Rayleigh-Brillouin spectrum of the clusterfree OTP is as predicted by the theories, in contrast to the experimental data for all other glass-forming liquids. Thus the cluster-free OTP represents a nonequilibrium state of the supercooled liquid. The equilibration of the cluster-free OTP may not be possible for different reasons: (i) The energy difference between the equilibrium (with clusters) and nonequilibrium (cluster-free) forms of OTP may be low, (ii) long-range correlations are hindered, or (iii) in samples with clusters even above $T_{m}$ some short-range correlations are left, which lead to cluster formation upon cooling. These short-range correlations may not be present in a cluster-free sample. The equilibration time, measured for BMMPC, is 11 orders of magnitude longer than the relaxation time of the $\alpha$ process, thus it is impossible to equilibrate the glass-forming liquids in terms of the long-range density fluctuations close to $T_{g}$.

\section{ACKNOWLEDGMENT}

The authors gratefully acknowledge the partial financial support of the Deutsche Forschungsgemeinschaft (SFB 262).
[1] H. Sillescu, J. Non-Cryst. Solids 243, 81 (1999).

[2] M.D. Ediger, C.A. Angell, and S.R. Nagel, J. Non-Cryst. Solids 100, 1300 (1996).

[3] K. Schmidt-Rohr, H.W. Spiess, Phys. Rev. Lett. 66, 3020 (1991).

[4] K. Schmidt-Rohr and H.W. Spiess, Multidimensional Solidstate NMR and Polymers (Academic Press, London, 1994).

[5] B. Schiener, R. Böhmer, A. Loidl, and R.V. Chamberlin, Science 274, 752 (1996).

[6] R. Richert, J. Phys. Chem. B 101, 6323 (1997); J. Non-Cryst. Solids 235-237, 41 (1998).

[7] M.T. Cicerone, F.R. Blackburn, and M.D. Ediger, J. Chem. Phys. 102, 471 (1995).

[8] C.Y. Wang and M.D. Ediger, J. Phys. Chem. B 103, 4177 (1999).

[9] I. Chang, F. Fujara, B. Geil, G. Heuberger, T. Mangel, and H. Sillescu, J. Non-Cryst. Solids 172-174, 248 (1994).

[10] E.R. Weeks, J.C. Crocker, A.C. Levitt, A. Schofield, and D.A. Weitz, Science 287, 627 (2000).

[11] G. Adams and J.H. Gibbs, J. Chem. Phys. 43, 139 (1965).

[12] E.J. Donth, J. Non-Cryst. Solids 53, 325 (1982).

[13] E. J. Donth, Relaxation and Thermodynamics in Polymers (Akademie Verlag, Berlin, 1992).

[14] U. Tracht, M. Wilhelm, A. Heuer, H. Feng, K. Schmidt-Rohr, and H.W. Spiess, Phys. Rev. Lett. 81, 2727 (1998).

[15] C. Bennemann, C. Donati, J. Baschnagel, and S.C. Glotzer, Nature (London) 399, 246 (1999).
[16] T. Tomita and T. Egami, Phys. Rev. B 52, 3290 (1995).

[17] D. Forster, Hydrodynamic Fluctuations, Broken Symmetry and Correlation Functions (Benjamin/Cummings Publ. Comp., London, 1975).

[18] R.D. Mountain, J. Res. Natl. Bur. Stand., Sect. A 70A, 207 (1966).

[19] C.J. Montrose, V.A. Solovyev, and T.A. Litovitz, J. Acoust. Soc. Am. 43, 117 (1968).

[20] Y.-H. Lin and C.H. Wang, J. Chem. Phys. 69, 1546 (1978).

[21] G.D. Patterson, Annu. Rev. Mater. Sci. 13, 219 (1983).

[22] K.L. Ngai, R.W. Rendel, and C.H. Wang, Polymer 30, 369 (1989).

[23] L. Börjesson, L.M. Torell, and J.R. Stevens, Polymer 30, 370 (1989).

[24] E.W. Fischer, Ch. Becker, J.-U. Hagenah, and G. Meier, Prog. Colloid Polym. Sci. 80, 198 (1989).

[25] C.H. Wang, X.R. Zhu, and J.C. Shen, Mol. Phys. 62, 749 (1987).

[26] H. Nilgens, M.Sc. thesis, Mainz (1991).

[27] E.W. Fischer, in Basic Features of the Glassy State, edited by J. Colmenero and A. Alegria (World Scientific, Singapore, 1990), p. 172.

[28] E.W. Fischer, G. Meier, T. Rabenau, A. Patkowski, W. Steffen, and W. Thönnes, J. Non-Cryst. Solids 131-133, 134 (1991).

[29] T. Kanaya, A. Patkowski, E.W. Fischer, J. Seils, H. Gläser, and K. Kaji, Acta Polym. 45, 137 (1994). 
[30] E.W. Fischer, Physica A 201, 183 (1993).

[31] A. Patkowski, H. Gläser, T. Kanaya, and E.W. Fischer (unpublished).

[32] W. Steffen, A. Patkowski, G. Meier, and E.W. Fischer, J. Chem. Phys. 96, 4171 (1992).

[33] W. Thönnes, Ph.D. thesis, Mainz (1990).

[34] E.W. Fischer, E. Donth, and W. Steffen, Phys. Rev. Lett. 68, 2344 (1992).

[35] A. Patkowski, Th. Thurn-Albrecht, E. Banachowicz, W. Steffen, P. Bösecke, T. Narayanan, and E.W. Fischer, Phys. Rev. E 61, 6909 (2000).

[36] E.W. Fischer, A.S. Bakai, in Slow Dynamics in Complex Systems, edited by Michio Tokuyama and Irwin Oppenheim, AIP Conf. Proc. No. 469 (AIP, Woodbury, 1999), p. 325.

[37] H. Tanaka, J. Phys.: Condens. Matter 11, L159 (1999).

[38] H. Tanaka, J. Chem. Phys. 111, 3163 (1999).

[39] W. Götze, in Liquids, Freezing and the Glass Transition, Les Houches Summer School, Session LI, edited by, D. Levesque, J.P. Hansen, and J. Zinn-Justin (Elsevier, Amsterdam, 1991).

[40] A. Patkowski, W. Steffen, H. Nilgens, E.W. Fischer, and R. Pecora, J. Chem. Phys. 106, 8401 (1997).

[41] R.L. Murry, J.T. Fourkas, W.-X. Li, and T. Keyes, Phys. Rev. Lett. 83, 3550 (1999).

[42] R.J. Greet and D. Turnbull, J. Chem. Phys. 46, 1243 (1967).

[43] B. Gerharz, G. Meier, and E.W. Fischer, J. Chem. Phys. 92, 7110 (1990).

[44] E. Eckstein, J. Qian, R. Hentschke, T. Thurn-Albrecht, W. Steffen, and E.W. Fischer, J. Chem. Phys. 113, 4751 (2000).

[45] T.M. Bender, R.J. Lewis, and R. Pecora, Macromolecules 19, 244 (1986).

[46] M. Baumann M.Sc. thesis, Mainz (1990).

[47] A. Patkowski, E.W. Fischer, H. Gläser, H. Nilgens, and W.
Steffen, Prog. Colloid Polym. Sci. 91, 35 (1993).

[48] J. Feder, Fractals (Plenum, New York, 1988), p. 36.

[49] A. Bakai and E.W. Fischer (unpublished).

[50] P. Debye, H.R. Anderson, Jr., and H. Brumberger, J. Appl. Phys. 28, 679 (1957).

[51] P. Tartaglia, J. Rouch, and S.H. Chen, Phys. Rev. A 45, 7257 (1992).

[52] B. Berne, and R. Pecora, Dynamic Light Scattering (Wiley, New York, 1976).

[53] R. Kubo, M. Toda, and N. Hashitsume, Statistical Physics II (Springer, Berlin, 1985).

[54] L. Reinhardt, E.W. Fischer, and A. Patkowski (unpublished).

[55] P. Debye and A.M. Bueche, J. Appl. Phys. 20, 518 (1949).

[56] M. Naoki and S. Koeda, J. Phys. Chem. 93, 948 (1989).

[57] Y. Higashigaki and C.H. Wang, J. Chem. Phys. 74, 3175 (1981).

[58] Y.Y. Huang and C.H. Wang, J. Chem. Phys. 61, 1868 (1974).

[59] W.L. Peticolas, G.L. Stegeman, and B.P. Stoicheff, Phys. Rev. Lett. 18, 1130 (1967).

[60] K.F. Herzfeld and T.A. Litovitz, Absorption and Dispersion of Ultrasonic Waves (Academic Press, New York, 1959).

[61] D.A. Jackson, H.T.A. Pentecost, and J.G. Powels, Mol. Phys. 23, 425 (1972).

[62] A. Aouadi, C. Dreyfus, M. Massor, R.M. Pick, T. Berger, W. Steffen, A. Patkowski, and C. Alba-Simionesco, J. Chem. Phys. 112, 9860 (2000).

[63] D.A. Pinnow, S.J. Candau, J.T. La Macchia, and T.A. Litovitz, J. Acoust. Soc. Am. 43, 131 (1968).

[64] G. D’Arrigio, J. Chem. Phys. 63, 61 (1975).

[65] D.W. Schaefer, J.E. Martin, P. Wiltzius, and D.S. Cannel, Phys. Rev. Lett. 52, 2371 (1984).

[66] J.E. Martin and A.J. Hurd, J. Appl. Crystallogr. 20, 61 (1987). 